\section{Loudness effects in pairs of tone bursts}

\author{
R. J. IRWIN* and J. J. ZWISLOCKI \\ Laboratory of Sensory Communication \\ Syracuse University, Syracuse, New York 13210
}

The loudness of dichotic and monotic pairs of short tone bursts was investigated as a function of the interburst time interval. For short intervals, the loudness was increased relative ty the loudness of a single burst. However, the loudness of a burst pair was equal to the loudness of the second burst in the pair and, therefore, no loudness summation but only a loudness enhancement took place. In dichotic bursts, the loudness enhancement jecayed monotonically as the time interval increased, and the rate of decay increased with sound intensity. In monotic bursts, the loudness enhancement decayed to a minimum at about $40 \mathrm{msec}$, independent of sound intensity. It had a tendency to rebound at longer time intervals and go through a relative maximum in the vicinity of $200 \mathrm{msec}$. The results are interpreted in terms of an interaction of peripheral and central poststimulatory inhibition with temporal summation.

Experiments with short pulses and tone bursts at the threshold of audibility (Garner, 1947; Garner \& Miller, 1947; Feldtkeller \& Oetinger, 1956; Zwislocki et al, 1962) led to a mathematical theory that predicts the threshold for practically any temporal pattern (Zwislocki, 1960). An extension of this theory has made it possible to describe quantitatively the growth of loudness with stimulus duration (Zwislocki, 1969). In order to extend the theory further to the loudness of intermittent stimuli, more empirical information is required than is available at present. For this reason, several series of experiments have been initiated in the Laboratory of Sensory Communication. The present article describes the first of them.

The experiments deal with loudness effects in monotic and dichotic pairs of tone bursts. In several respects, they resemble some of the experiments performed by Niese (1956) and by Schwarze (1963), and more recently by Scharf and his coworkers (Scharf, 1969a, b; Scharf \& Weissman, 1970). However, they illuminate the process involved from a different angle. Also, the procedures were different and have led to somewhat divergent results. We asked three fundamental questions: (1) What is the loudness level of a burst pair as a function of the interburst time interval? (2) Does the loudness of the second burst differ from the loudness of the first burst and from the loudness of the burst pair when both bursts have the same intensitv? (3) How does the dichotic time function differ from the monotic one? In addition, we explored the effects of sound pressure level and of frequency difference.

\section{METHOD \\ Apparatus}

The tone bursts were produced by

* On sabbatical from the University of Auckland, New Zealand. cutting out portions of a 1,000-Hz sine wave from a Hewlett-Packard oscillator (Model 201CR) in such a way as to keep their frequency spectrum substantially within one critical band (about $170 \mathrm{~Hz}$ ). They were turned on and off by Grason Stadler electronic switches with a rise and decay time of $5 \mathrm{msec}$. The bursts were attenuated and fed to matched earphones (TDH 39) by General Radio amplifiers (Model 1206-B) and matching resistance pads.

Three electronic switches produced three bursts of 10-msec duration between half power points and of identical shape, as judged from their display on an oscilloscope. The first two bursts were separated by a variable interval that was controlled by Tektronix equipment (Types 161 and 162) and measured by an electronic counter (Hewlett-Packard, Model 522B). These two bursts could either be presented dichotically or mixed in an amplifier and presented monotically. The third burst always went to the same ear as the second burst and always followed it by 500 msec. The burst triplet was recycled every 2 sec. The sound pressure level of the bursts was specified by measuring the voltage across the earphone for a $1,000-\mathrm{Hz}$ tone of long duration and by converting to SPLs from the calibration of the earphone in a 6 -cc coupler, with the help of Jerger and Tillman's (1959) correction table. The sound pressure level so specified coincided with the peak sound pressure of the bursts.

\section{Procedure}

The listener sat in a soundproofed booth and wore earphones mounted in circumaural cushions CZW6 (Zwislocki, 1955). He was asked to match the loudness of the third burst to the loudness of either the first burst or the second, or to the overall loudness of both bursts. He performed his task by turning an unmarked knob that controlled the intensity of the third burst in 2-dB steps until he was satisfied with the match. He was instructed to "bracket" the reference loudness.

When the first two bursts were presented to different ears, they were set equal in loudness with the help of separate matches with the third burst. When they were presented to the same ear, they were set at an equal SPL. The experiment began by matching the third burst to the second presented alone. This provided the reference level for loudness-level changes produced by interburst interactions. During each experimental session, the interval between the first two bursts was systematically increased and decreased, the initial direction being determined at random, until a $S$ had made four judgments at each interval. The temporal loudness functions were investigated at three SPLs, 30,60 , and $90 \mathrm{~dB}$. One SPL was used per session, according to a random order.

\section{Subjects}

The data reported here are from 15

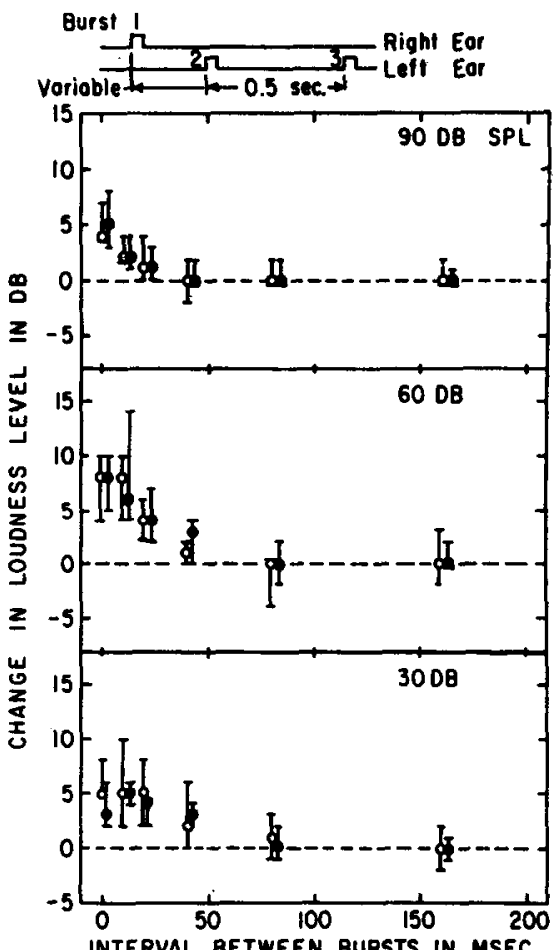

INTERVAL BETWEEN BURSTS IN MSEC.

- Overoll loudness level of bursts 1 and 2

- Loudness level of bursl 2

Fig. 1. Change in loudness level of a dichotic burst pair and of the second burst in the pair as a function of the interburst time interval; both bursts at $1,000-\mathrm{Hz}$ sound frequency. The circles indicate the medians and the vertical bars the interquartile ranges of 16 judgments (four listeners). 


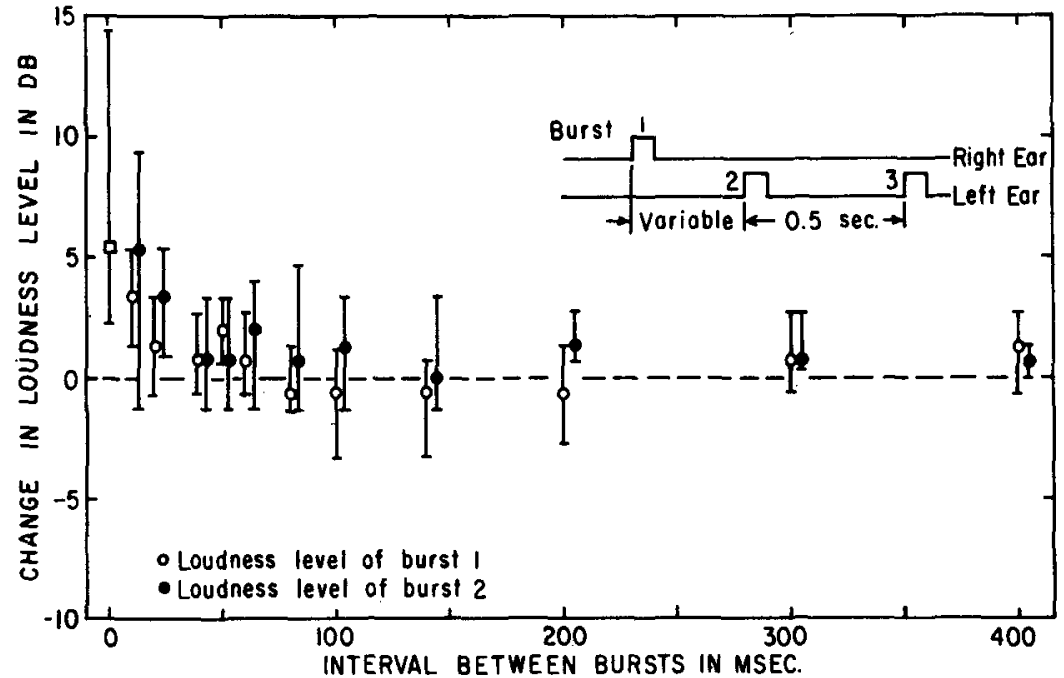

Fig. 2. Changes in loudness levels of the first and second burst of a dichotic burst pair as a function of the interburst time interval; both bursts at $1,000 \mathrm{~Hz}$ and $40 \mathrm{~dB}$ SL. The circles indicate the medians, and the vertical bars the interquartile ranges of 21 judgments (seven listeners). The square shows the median overall loudness of both bursts presented simultaneously (10 judgments by each of six listeners).

Ss with normal hearing, including the authors. Some Ss served in only one task, some in several. All had at least one session of practice. The task proved to be difficult, and some potential Ss were not used because their judgments were too variable, despite several hours of practice.

\section{Dichotic Stimuli}

\section{RESULTS}

Figure 1 shows the loudness level of the dichotic burst pair (unfilled circles) and of the second burst in the pair as a function of the interburst time interval. The first set of data was obtained by asking the listeners to match the loudness of the third burst to the overall loudness of the pair; the second set, by asking them to match the loudness of the third burst to that of the second. The ordinate zero corresponds to the loudness level of the second burst presented alone. Each graph corresponds to a different reference SPL of 30,60 , and $90 \mathrm{~dB}$, respectively. The stimulus time pattern is indicated at the top of the figure.

Perhaps the most fundamental result evident from Fig. 1 is the loudness equality between the burst pair and the second burst, independent of the time interval and SPL. Such equality is not surprising at short time intervals at which the two bursts are fused. But it persisted at intervals longer than $20 \mathrm{msec}$, at which the bursts were heard separately. Thus, it appears that listeners were unable to integrate the loudness of a burst pair and that the total loudness was determined by the loudness of the second burst. In view of this finding, it may be more accurate to ascribe the from that in Fig. 1. The unfilled circles indicate the loudness level of the first burst and the closed circles, that of the second relative to the loudness level of a burst presented alone. The loudness level of the second burst follows the same decay pattern as in Fig. 1. The loudness level of the first burst seems to be consistently lower. Because this tendency persists at very short time intervals, at which the bursts are fused, it is not clear whether the difference is real or due to an experimental artifact. The smallness of the loudness enhancement and a substantial variability of the data precludes a definitive decision. Since the problem is of fundamental importance, further investigations will be undertaken.

Note that Fig. 2 contains only one point at the zero time interval, that is, for complete burst coincidence. This point was obtained on a separate occasion from the other time intervals, and the listeners were asked simply to match the loudness of the monaural burst to that of the binaural one. The ordinate of the point is consistent with the values of Fig. 1.

increased loudness to loudness enhancement rather than to loudness summation.

Another result shown in Fig. 1 is the rapid time decay of the loudness enhancement, particularly at high SPLs. It is substantially more rapid than we would have predicted from the known time constant of temporal auditory summation, which is on the order of $200 \mathrm{msec}$ (Feldtkeller \& Oetinger, 1956; Zwislocki, 1960, 1969). However, the decay is more gradual at low SPLs, as shown in the lowest graph for a SPL of $30 \mathrm{~dB}$. Near the threshold of audibility, it probably becomes consistent with the 200-msec time constant, in agreement with earlier dichotic threshold experiments (Zerlin, 1966; Schenkel, 1967).

There is one more feature of the data in Fig. 1 worthy of notice. It concerns the magnitude of the loudness-level change as a function of SPL. In agreement with the results of Scharf (1969a), the greatest effect occurs at medium SPLs and decreases at high and low levels. As a consequence, medium SPLs appear as the most appropriate for loudness investigations on sequences of tone bursts.

Additional information on loudness relationships in dichotic pairs of tone bursts was obtained by means of experiments in which listeners were asked to match the loudness of the third burst to that of either the first or the second burst. These experiments were performed on a different group of seven listeners at a sensation level of $40 \mathrm{~dB}$, which approximates a SPL of $50 \mathrm{~dB}$. The results are shown in Fig. 2. Note that the time scale is different

\section{Monotic Stimuli}

In these experiments, all three tone bursts were presented to one ear at three SPLs, of 30,60 , and $90 \mathrm{~dB}$. In the first two series, all bursts were at the same sound frequency of $1,000 \mathrm{~Hz}$. The listeners matched the loudness of the third burst to the overall loudness of the preceding burst pair. Four listeners participated in the first series, and the interburst time interval varied between 0 and $160 \mathrm{msec}$. In a second series, with two listeners, the time interval covered the range between 60 and $500 \mathrm{msec}$. The results are shown in Fig. 3. The circles belong to the four-listener series, the squares to the two-listener one. The results of both series are mutually consistent and indicate a somewhat different loudness-level pattern than in the dichotic experiments. At short intervals, the loudness level rapidly decays to that of a single burst, and a minimum level is reached between 20 and $60 \mathrm{msec}$. Subsequently, the loudness rebounds and reaches a relative maximum in the vicinity of $200 \mathrm{msec}$. This pattern is clearly evident at 60-dB SPL, somewhat less so at $90 \mathrm{~dB}$, and is barely perceptible at $30 \mathrm{~dB}$. It probably stems from a complexity of processes. At zero time interval, a physical stimulus summation takes place. Because of phase equality, it should produce a 6-dB enhancement of loudness level, corresponding to a 6 -dB enhancement of SPL. Because of the 10-msec burst duration and the 5-msec onset and decay times, a partial physical summation persists up to about 

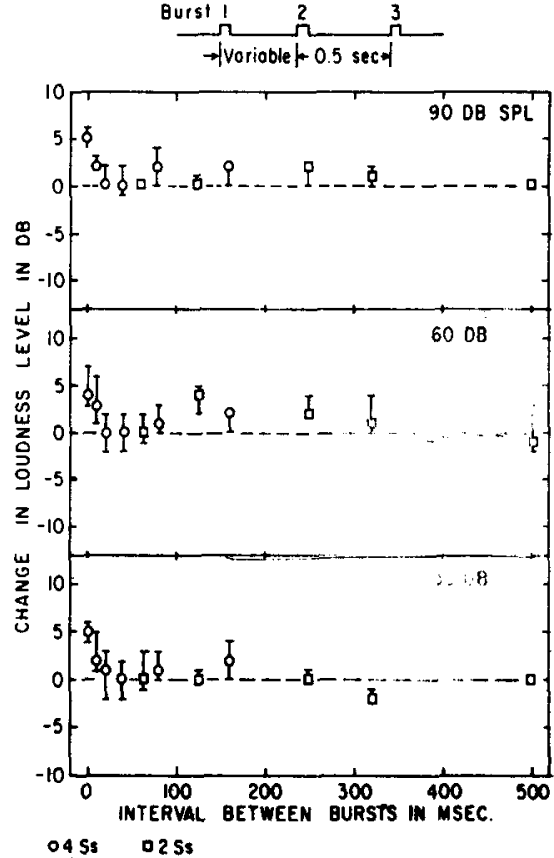

Fig. 3. Change in loudness level of a monotic burst pair as a function of the interburst time interval. The circles indicate the medians of 16 judgments (four listeners), and the squares, the medians of 8 judgments (two listeners). The vertical bars show the interquartile ranges.

20 msec. As a consequence, the increased loudness level at short time intervals may be partially explained by the summation of stimulus amplitudes. At longer intervals, the loudness level is probably determined by an interaction of summation and inhibition processes in the nervous system. Scharf (1969b) has demonstrated that the inhibitory effect may be decreased by making the sound frequency of the second burst considerably different from that of the first. Under these conditions, the loudness level may be increased by as much as $10 \mathrm{~dB}$, and it decays monotonically with the increasing time interval.

In order to check the effect of frequency difference under our experimental conditions, we increased the sound frequency of the first burst to $2,100 \mathrm{~Hz}$, leaving the sound frequency of the remaining two bursts at $1,000 \mathrm{~Hz}$. The second burst was set at $60 \mathrm{~dB}$ SPL, and the first was adjusted to loudness equality with it. Two listeners were involved in the experiment. The results are shown in Fig. 4 by means of filled circles and compared to the data of Fig. 1 for $60 \mathrm{~dB}$ SPL, the latter indicated by the unfilled circles. Clearly, the frequency difference increased the loudness level, in agreement with Scharf's results. This is particularly true around
$40 \mathrm{msec}$, where the loudness minimum completely disappeared.

\section{DISCUSSION}

Perhaps the most important finding of this investigation is that, under our experimental conditions, listeners are unable to integrate the loudness of two-burst sequences. Whether they are asked to match the overall loudness of a pair of tone bursts or the loudness of the second burst in a pair, the result is the same even when the bursts can be heard separately. If a temporal integration of loudness took place, the overall loudness would have to exceed the loudness of either burst alone. As we found out under similar experimental conditions, the loudness of the first burst is not substantially different from that of the second. As a consequence, a complete integration would make the loudness of the burst pair appear nearly twice the loudness of the second burst. In the absence of an actual loudness summation, it may be more appropriate to speak of loudness enhancement instead of summation when two bursts are judged to be louder than a burst presented alone.

Our results indicate that loudness effects in dichotic or monotic pairs of tone bursts cannot be explained in terms of the simple temporal summation that appears to control the threshold of audibility for various stimulus time patterns (Zwislocki, 1960). The time constant of this summation is on the order of $200 \mathrm{msec}$ and would produce an exponential decay of loudness enhancement over a comparable time span. The empirical data indicate that the enhancement disappears within less than $100 \mathrm{msec}$ in dichotic burst pairs and follows a nonmonotonic course in monotic pairs. Furthermore, the duration of the dichotic enhancement decreases as the intensity level increases. It would be possible to account for these results by postulating a dichotic summation mechanism with a time constant dependent on sound intensity and a different monotic mechanism. However, such a model would not go beyond rudimentary curve fitting. A tentative model of much wider generality may be derived by taking into consideration additional psychoacoustic and neurophysiological data.

Scharf (1969b) has shown that, when the sound frequency of the first burst is sufficiently different from that of the second, the loudness enhancement persists over more than $200 \mathrm{msec}$, and its magnitude is compatible with simple loudness summation. Our results with two bursts of different frequency are

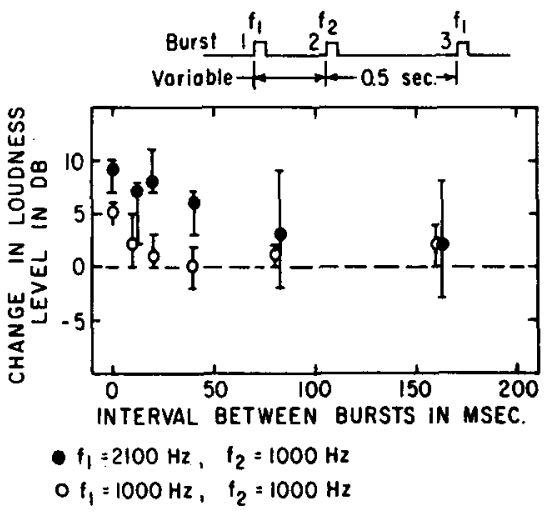

Fig. 4. Change in loudness level of a monotic burst pair as a function of the interburst time interval when the sound frequency of the first burst is different from that of the second. The medians of 8 judgments (two listeners) are indicated by the filled circles and the interquartile ranges by the vertical bars. For comparison, the unfilled circles reproduce the data of the middle graph in Fig. 3, which shows the loudness-level change when both bursts have the same sound frequency.

consistent with Scharf's finding. As a consequence, it appears reasonable to assume that the same temporal summation that manifests itself at the threshold of audibility also affects the loudness of burst pairs. Its decreased effect, when both bursts have the same sound frequency, may be accounted for by assuming poststimulatory inhibition. Such inhibition is well known from neurophysiological experiments on peripheral and central portions of the auditory system. When two clicks of the same amplitude are presented to the same ear, the eighth nerve response to the second click is depressed over a period of about 50 msec (Rosenzweig \& Rosenblith, 1953). The evoked and single unit response in the medial geniculate body and in the primary cortical projection area may be depressed over more than 200 msec (Rosenzweig \& Rosenblith, 1953; Parker \& Mundie, 1967; Aitkin \& Dunlop, 1968; Etholm, 1969). These poststimulatory effects appear to be frequency selective, and the ones with the long time constant develop with an appreciable latency. An interaction of temporal summation with the slow poststimulatory inhibition that manifests itself in the medial geniculate body and the cortex is consistent with the moderately rapid decay of loudness enhancement in dichotic burst pairs. An acceleration of the decay in monotic burst pairs could be accounted for by the peripheral poststimulatory inhibition. The slight rebound of loudness around $200 \mathrm{msec}$ could be due to postinhibitory sensitization sometimes seen in 
single-unit recordings (Starr \& Britt, $1970 ;$ Smith $^{1}$ ).

While our data agree with Scharf's (Scharf, 1969b; Scharf \& Weissman, 1970 ) in principle, some systematic numerical discrepancies are evident. In dichotic burst pairs, we have found a somewhat faster loudness decay than he did. The difference may stem from a slightly different stimulus configuration. Scharf used 5-msec bursts with $1.5-\mathrm{msec}$ rise and fall times as opposed to our 10 -msec bursts with 5-msec rise and fall times. As a result, his spectral bandwidth was greater than ours and probably exceeded the critical band. From Scharf's and Weissman's (1970) results, it appears that the loudness enhancement decays more slowly for broad-band stimuli than for narrow-band ones. There is still another difference in the stimulus configuration. While they used a diotic third burst, we used a monotic one. In monotic burst pairs, Scharf's (1969) data do not indicate a clear loudness minimum at a time separation of about $40 \mathrm{msec}$ as ours do. Nevertheless, a flattening of the decay curves in this region is evident. Not all our curves exhibited a clear minimum, and the effect is so small that it can easily hide in the scatter of the data. Thus, we may conclude that Scharf's and our data are mutually consistent.

\section{REFERENCES}

AITKIN, L. M., \& DUNLOP, C. W.
Inhibition in the medial geniculate body of the cat. Experimental Brain Research. $1969,7,68-83$.

ETHOLM, B. Evoked responses in the inferior colliculus, medial geniculate body and auditory cortex by single and douive clicks in cats. Acta Oto-Laryngologica. $1969,67,319-325$.

FELDTKELLER, $R$., \& OETINGER, $R$. Die Hörbarkirtsgrenzen von Impulsen verschiedener Daner. Acoustica Akust. Beih., 1956, 6, 489-493.

GARNER, W. R. Auditory thresholds for short tones as a function of repetition rate. Journal of the Acoustical Society of America, 1947, 19,600-608.

GARNER, W. R., \& MILLER, G. A. The masked threshold of pure tones as a function of duration. Journal of Experimental Psychology, 1947, 37, 293-303

JERGER, J. F., \& TILLMAN, T. W. Effect of earphone cushion on auditory threshold. Journal of the Acoustical Society of America, 1959, 31, 1264-1265.

NIESE, H. Vorschlag für die Definition und Messung der Deutlichkeit nach s u b j e k t i ven Grundlagen. Hochfrequenztechnikn. und Elektroak. $1956,65,4-15$ (cit. Scharf, 1969b)

PARKER, D. E., \& MUNDIE, J. R. Neural sensitivity changes following stimulation with transient sound bursts. Journal of Auditory Research, 1967, 7, 287-301.

ROSENZWEIG, $M$. R \& ROSENBLITH W. A. Responses to auditory stimuli at the cochlea and at the auditory cortex. Psychological Monographs, 1953, 67 363-389.

SCHARF, B. Dichotic summation of loudness. Journal of the Acoustical Society of America, 1969a, 45 . 1193-1205.

SCHARF, B. Loudness and frequency selectivity at short durations. Proceedings of the Symposium on Frequency Analysis and Periodicity Detection in Hearing,
$1969 \mathrm{~b}$, Driebergen, The Netherlands.

SCHARF, B.. \& WEISSMAN, S. M. Dichotic summation of loudness over time. Journal of the Acoustical Society of America, $1970,47,96(\mathrm{~A})$.

SCHENKEL, K. D. Die beidohrigen Mithörschwellen von Impulsen. Acustica, $1967,18,38-46$.

SCHWARZE, D. Die Lautstärke von Gausstönen. Doctoral dissertation, Technischen Universität, Berlin, 1963 (cit. Scharf, 1969b).

STARR, A., \& BRITT, R. Intracellular recordings from cat cochlear nucleus during tone stimulation. Joumal of Neurophysiology, 1970, 33, 137-147.

ZERLIN, S. Interaural time and intensity differences and the MLD. Journal of the Acoustical Society of America, 1966, 39, 134-137.

ZWISLOCKI, J. Development of a semi-plastic earphone socket. Journal of the Acoustical Society of America, 1955. 27, 155-161.

ZWISLOCKI, J. Theory of temporal auditory summation. Journal of the Acoustical Society of America, 1960, 32, 1046-1060.

ZWISLOCKI, J. J. Temporal summation of loudness: An analysis. Journal of the Acoustical Society of America, 1969, 46, 431-441.

ZWISLOCKI, J., HELLMAN, R. P., \& VERRILLO, R. T. Threshold of audibility for short pulses. Journal of the Acoustical Society of America, 1962, 34 . 1648-1652.

\section{NOTE}

1. R. L. Smith, personal communication, 1970 .

(Accepted for publication February 24, 